\title{
Thermally induced core-electron binding-energy shifts in transition metals: An experimental investigation of $\mathrm{Ta}(100)$
}

\author{
D. M. Riffe \\ Department of Physics, Utah State University, Logan, Utah 84322-4415 \\ W. Hale, B. Kim, ${ }^{*}$ and J. L. Erskine \\ Department of Physics, University of Texas, Austin, Texas 78712
}

(Received 15 August 1996)

\begin{abstract}
High-resolution photoemission spectra from the $4 f_{7 / 2}$ levels of $\mathrm{Ta}(100)$ have been obtained between $77 \mathrm{~K}$ and room temperature. The data show an increase in both the surface and bulk core-level binding energies (BE's) as the temperature is raised: between 77 and $293 \mathrm{~K}$ the bulk and surface BE's increase by $31 \pm 3$ and $13 \pm 2 \mathrm{meV}$, respectively. A model calculation of the bulk binding-energy increase, which is based upon the lattice expansion of the solid, is in good agreement with the experimental results and indicates that the shifts arise from both initial- and final-state effects that are of comparable magnitude. The model is further used to estimate thermally induced shifts for the whole $5 d$ transition-metal series. [S0163-1829(96)02348-X]
\end{abstract}

\section{INTRODUCTION}

Temperature-induced changes in core-level binding energies (BE's) provide a unique perspective on the electronic structure of a solid-state system. This is because the thermally induced BE shifts are directly related to the thermally induced expansion of the solid. In ionic solids, for example, the BE changes arise from expansion-induced changes in the Madelung potential, which perturbs the BE's of cation and anion core levels in opposite directions. ${ }^{1}$ In simple metals, such as the alkali metals $\mathrm{Li}, \mathrm{Na}, \mathrm{K}$, and $\mathrm{Rb},{ }^{2-4}$ alkaline earths $\mathrm{Be}$ and $\mathrm{Mg}$,, 3 and trivalent metals $\mathrm{Al}$ and $\mathrm{In},{ }^{2,4,5}$ the thermal shifts have been shown to arise from changes in the conduction-band electronic structure in both the initial and final states of the photoemission process. ${ }^{4}$ For these simple metals, the thermally induced changes in BE's have provided insight into a microscopic description of their surface corelevel shifts (SCS's). ${ }^{4}$ In contrast to the simple metals, $d$-band transition metals have received little attention ( $\mathrm{Lu}$ appears to be the only case ${ }^{6}$ ) with regard to thermally induced changes in core-level BE's. This is somewhat surprising, given the vast experimental work on the SCS's in these materials. ${ }^{7}$ Since a theory of transition-metal SCS's is well established, 7,8 it is worth investigating thermally induced shifts in order to see if they can be described within the same theoretical framework.

Here we report on a temperature-dependent study of Ta(100) core-level BE's. The thermal shifts in the bulk and surface atoms are both positive but are measurably different, in contrast to results for the alkali metals where bulk and surface thermal shifts are identical. ${ }^{4}$ The measured shifts agree reasonably well in both sign and magnitude with a simple theoretical model that is based on a combination of the standard initial-state model of transition-metal SCS's (Ref. 8) and a final-state contribution from changes in conduction-electron screening of the core hole. ${ }^{4}$ Predictions for thermally induced BE shifts across the $5 d$ transitionmetal series are provided as an impetus for more experimental work in this area.

\section{EXPERIMENTAL DETAILS}

The core-level data were obtained on the University of Texas/Sandia beam line on the vacuum ultraviolet ring at the National Synchrotron Light Source at Brookhaven National Laboratory. Photon energies of 70 and $100 \mathrm{eV}$ were used to collect data at a total resolution of $130 \pm 10 \mathrm{meV}$.

The $\mathrm{Ta}(100)$ crystal $(1 \mathrm{~cm}$ diameter by $0.1 \mathrm{~cm}$ thick) was oriented (to within $\pm 0.5^{\circ}$ ), spark cut, and mechanically polished. The crystal was cleaned in situ by repeated annealing in a base-pressure vacuum of $8 \times 10^{-11}$ Torr to $2850 \mathrm{~K}$ (Ref. 9) until the core-level spectra exhibited no contamination features and could be well fit with Doniach-Šnjic line shapes ${ }^{10}$ for all components. ${ }^{11}$

Each set of temperature-dependent spectra was obtained as follows. First, the sample was cooled to $77 \mathrm{~K}$ via a $\mathrm{Cu}$ cold finger attached to the bottom of a liquid- $\mathrm{N}_{2}$ reservoir. After emptying the reservoir of liquid $\mathrm{N}_{2}$, the sample was quickly flashed to $\sim 2800 \mathrm{~K}$ in order to remove any contamination. The sample was then allowed to cool to a base temperature and a photoemission spectrum was then collected. The sample was then reflashed and recooled and the next spectrum subsequently collected. Since the $N_{2}$ reservoir was empty, the base temperature for the second spectrum was slightly above the temperature of the first. This three-step flash, cool, and measure process was repeated until the base temperature of the sample reached approximately room temperature. The large thermal mass of the cold finger allowed approximately 15 measurements to be made between $77 \mathrm{~K}$ and room temperature. After the highest temperature spectrum was collected the sample was cooled back down to 77 $\mathrm{K}$ and a photoemission spectrum was taken (after flashing and recooling) in order to check for any long-term systematic drift in the electronics that might have falsely produced an apparent temperature dependence in the binding energies.

\section{RESULTS}

Figure 1 shows a temperature-dependent series of $4 f_{7 / 2}$ core-level photoemission spectra from the $\mathrm{Ta}(100)$ sample. 


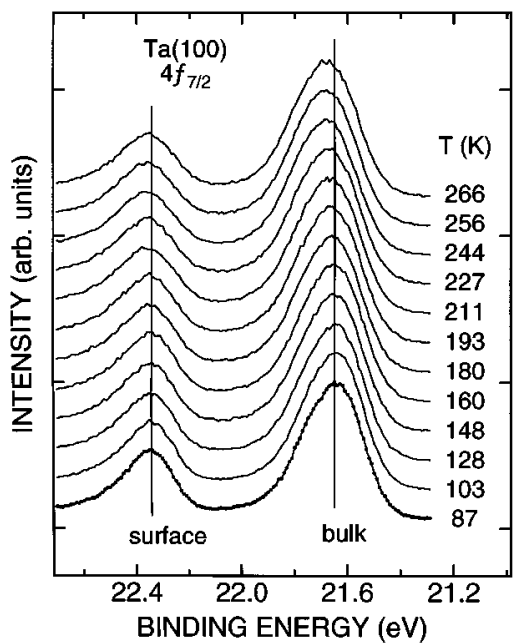

FIG. 1. $4 f_{7 / 2}$ core-level spectra of $\mathrm{Ta}(100)$ vs temperature. The vertical lines mark the low-temperature positions of each peak.

The higher BE peak (surface) arises from the first atomic layer of Ta atoms while the lower BE peak (bulk) comes from atoms in the second layer and deeper. The results of this study can be seen via inspection of this figure. As the temperature increases, the BE's (which for metals are referenced to the Fermi level) of both the bulk and surface peaks increase, with the bulk-atom BE increase substantially larger than that of the surface atoms.

A quantitative assessment of the temperature dependence of the BE's was carried out with a least-squares analysis of the data. A detailed description of the core-level fitting procedure of $\mathrm{Ta}(100)$ has been previously published. ${ }^{11}$ Briefly, the $\mathrm{Ta}(100) 4 f_{7 / 2}$ spectrum consists of three separate components, a single component for the surface feature and two components for the bulk, one due to the second atomic layer of atoms (underlayer) and one due to the rest of the bulk atoms. ${ }^{12}$ The underlayer and deeper bulk components are unresolved, but have been shown to be separated by $125 \pm 5 \mathrm{meV}$ at $80 \mathrm{~K} .{ }^{11}$ In fitting the temperature-dependent spectra, this underlayer shift was fixed at its low-temperature value. Each component in the spectrum was modeled with a Doniach-Š unjić (DS) line shape ${ }^{10}$ convolved with a Gaussian function. The DS line shape is described by two parameters, the singularity index $\alpha$ and a Lorentzian width $\Gamma$. Both $\alpha$ and $\Gamma$ were held fixed ${ }^{13}$ in the analysis to their lowtemperature values. ${ }^{11}$ The Gaussian broadening parameter was allowed to vary with temperature in order to account for the increase in phonon broadening with temperature.

Figure 2 plots the BE shifts (obtained from the leastsquares analysis) versus temperature. Results from the data in Fig. 1 as well as the results from fitting other data are plotted. Parts (a) and (b) show the shifts in BE for the bulk and surface peaks, respectively. The bulk-peak BE changes by $\sim 30 \mathrm{meV}$ between $77 \mathrm{~K}$ and room temperature, while the surface-peak shift is $\sim 10 \mathrm{meV}$. The temperature-dependent surface core-level shift, which is the difference in BE between atoms deep in the bulk and atoms in the first atomic layer is shown in Fig. 2(c). The SCS decreases by $\sim 20 \mathrm{meV}$ between 77 and $293 \mathrm{~K}$.

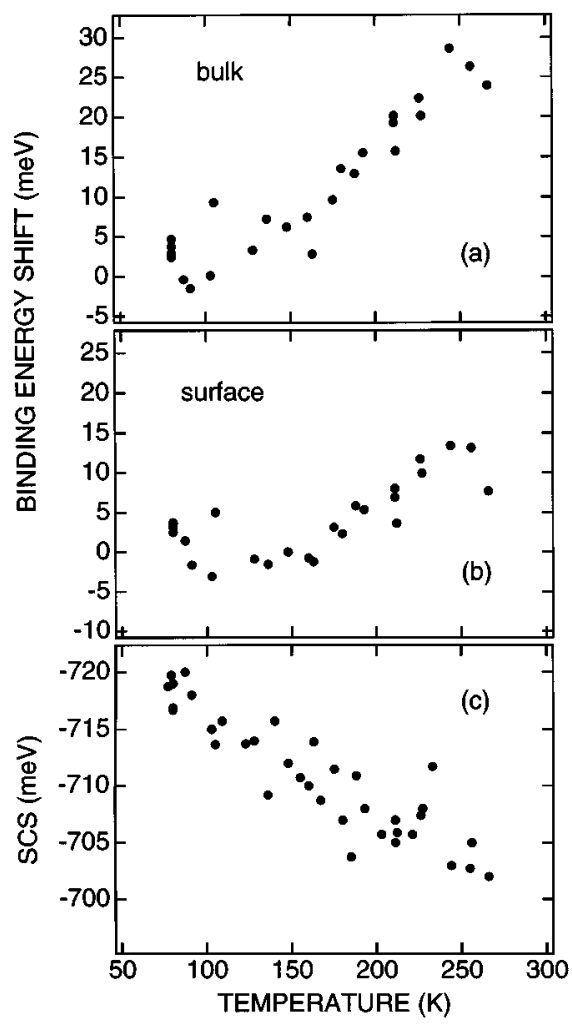

FIG. 2. Binding-energy shifts for the bulk (a) and surface (b) $4 f_{7 / 2}$ peaks of $\mathrm{Ta}(100)$ vs temperature. Also shown is the $\mathrm{Ta}(100)$ SCS (c) vs temperature.

\section{DISCUSSION}

For the alkali metals ${ }^{4}$ and the simple metal $\mathrm{Al}$ (Ref. 5) the temperature-dependent core-level BE shifts have been observed to be proportional to the linear thermal expansion $\Delta a / a$ of the solid. These observations motivate plotting the BE shifts of Ta versus $\Delta a / a$ for $\mathrm{Ta},{ }^{14}$ as shown in Fig. 3 . Although there is a fair amount of scatter in the data points, the data are consistent with a linear dependence upon $\Delta a / a$. Hence, we consider changes in the electronic structure of Ta as a function of the lattice constant $a$.

The most significant expansion-induced change in the valence-band electronic structure of a transition metal is the narrowing of the width $W_{d}$ of the valence $d$ band of the metal (the $5 d$ band for Ta). For a transition metal that has a less than half-filled $d$ band (as in the case of Ta) the centroid $E_{d}$ of the $d$ band necessarily decreases in energy (with respect to the Fermi level $E_{F}$ ) as the lattice constant increases. This is illustrated in Fig. 4(a), where for simplicity the $n s$ part of the valence-band density of states (DOS) is assumed to be free-electron-like $\left(\propto E^{1 / 2}\right)$ while the $(n-1) d$ DOS is constant over a finite region defined by the width $W_{d}$. Figure 4(b) illustrates the change in $E_{d}$ when the $d$ band is more than half filled. In this case $E_{d}$ increases in energy with respect to the $E_{F}$ as the lattice expands. This simplified DOS model, known as the Friedel model, ${ }^{15}$ is used below to calculate the initial-state contribution to the measured core-level shifts.

The change in the difference between $E_{d}$ and $E_{F}$ is the key ingredient in the initial-state contribution to the ther- 


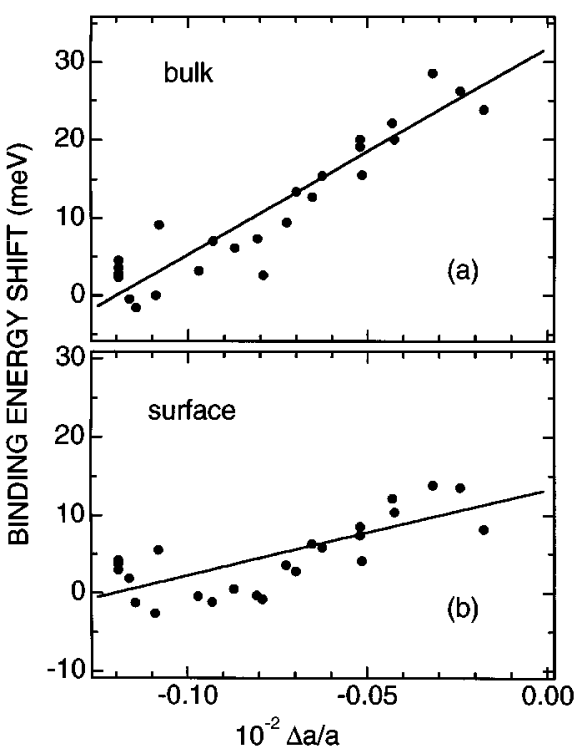

FIG. 3. Binding-energy shifts for the bulk (a) and surface (b) $4 f_{7 / 2}$ peaks of $\mathrm{Ta}(100)$ vs linear thermal expansion. The solid lines are linear least-squares fits through the experimental data points.

mally induced shifts in core-level BE's. This is because, with respect to the Fermi level, the core-electron energy levels essentially follow the centroid $E_{d}$ of the $d$ band. ${ }^{8}$ This has been borne out in tight-binding calculations of core-level shifts in a variety of transition metals ${ }^{7}$ and is the basis for the standard model of SCS's in transition metals. ${ }^{8}$ From the tight-binding calculations it has been shown that the corelevel shifts are $\sim 10 \%$ larger than the $d$-band centroid shifts.

The Friedel model in combination with a linear combination of atomic orbitals (LCAO) calculation of the bandwidth $W_{d}$ (Ref. 16) provides a simple expression for the change in $E_{d}-E_{F}$ as a function of $\Delta a / a$. As can be deduced from inspection of Fig. 4, the Friedel model relates $E_{d}-E_{F}$ to $W_{d}$ via

$$
E_{d}-E_{F}=\frac{W_{d}}{10}\left(5-Z_{d}\right),
$$

where $Z_{d}$ is the number of $d$ electrons per atom in the solid. The LCAO result for the bandwidth as a function of the atomic radius $r_{0}$ (Ref. 17) is given by ${ }^{16}$

$$
W_{d}=\frac{C}{r_{0}^{5}},
$$

where $C$ is a transition-metal-dependent constant. Using Eqs. (1) and (2) to calculate $\Delta\left(E_{d}-E_{F}\right) / \Delta\left(r_{0}\right)$ and substituting $\Delta a / a$ for $\Delta r_{0} / r_{0}$ yields the first-order expression for the change in $E_{d}-E_{F}$ versus $\Delta a l a$ as ${ }^{18}$

$$
\Delta\left(E_{d}-E_{F}\right)=-\frac{W_{d}\left(5-Z_{d}\right)}{2} \frac{\Delta a}{a} .
$$

As qualitatively discussed above in conjunction with Fig. 4, this expression shows for a less than half-filled $d$ band

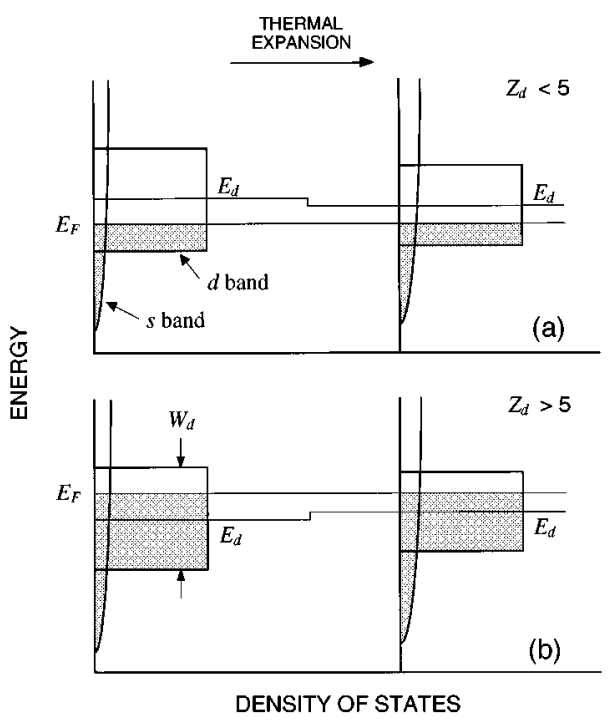

FIG. 4. Friedel model DOS illustration of the effect of lattice expansion on $E_{d}-E_{F}$ for a $d$-band transition metal. Both $Z_{d}<5$ (a) and $Z_{d}>5$ (b) cases are illustrated. See text for details.

$\left(Z_{d}<5\right)$ that $E_{d}-E_{F}$ decreases as the lattice expands. For the initial-state contribution $\Delta E_{\text {core }}^{i}$ to the core-level BE shift we thus have

$$
\Delta E_{\mathrm{core}}^{i}=1.1 \frac{W_{d}\left(5-Z_{d}\right)}{2} \frac{\Delta a}{a} .
$$

The change in sign between Eqs. (3) and (4) comes about because a shift in $\mathrm{BE}$ is equal but opposite to a shift in energy of the state.

There is also a final-state contribution $\Delta E_{\text {core }}^{f}$ to the measured core-level BE shifts. This contribution arises because the lattice expansion decreases the relaxation energy $E_{\text {relax }}$ associated with conduction-electron screening of the core hole. For simple metals the final-state contribution to the BE shift between the atomic and solid states, which arises from this conduction-electron screening, has been previously approximated using ${ }^{4}$

$$
E_{\text {relax }}=\frac{21.22}{\left(r_{s} / a_{0}\right)^{1 / 2}} \frac{1}{\left[1+0.815\left(r_{s} / a_{0}\right)^{1 / 2}\right]^{1 / 2}} \mathrm{eV},
$$

where $a_{0}$ is the Bohr radius, $r_{s}$ is given by $V=\frac{4}{3} \pi r_{s}^{3}$, and $V$ is the volume per conduction electron. $E_{\text {relax }}$ as given by Eq. (5) agrees very well with Hartree-Fock calculations ${ }^{19}$ of extra-atomic relaxation energies in simple metals. ${ }^{4}$ By differentiating Eq. (5) with respect to $r_{s}$, the final-state contribution $\Delta E_{\text {core }}^{f}$ to the core-level $\mathrm{BE}$ shift is calculated to be

$$
\Delta E_{\text {core }}^{f}=\frac{10.61}{\left(r_{s} / a_{0}\right)^{1 / 2}} \frac{\left[1+1.222\left(r_{s} / a_{0}\right)^{1 / 2}\right]}{\left[1+0.815\left(r_{s} / a_{0}\right)^{1 / 2}\right]^{3 / 2}} \frac{\Delta a}{a} \mathrm{eV},
$$

where again, the sign has been changed because a decreased relaxation energy results in increased core-electron BE's. 
Although Eqs. (5) and (6) were derived for simple metals, they appear to be reasonable approximations even for $d$-band transition metals as long as $r_{s}$ in each expression is obtained from the $s$-band charge density. Support for such use of these equations comes from recent numerical calculations, which indicate that in the $d$-band transition metals it is the $s$-band charge density that does most of the core-hole screening. ${ }^{20}$ Those calculations, which were done for a variety of transition metals, show that the $d$-electron contribution to the core-hole relaxation energy is typically only $20-40 \%$ of the total relaxation energy. In fact, when applied to the $3 d$ transition-metal series, Eq. (5) reproduces the slight increase in relaxation energy across the series ${ }^{21,22}$ and produces values for $E_{\text {relax }}$ that are, on average, only $25 \%$ larger than the relaxation energies calculated by Williams and Lang using a density-functional formalism. ${ }^{22}$

We now compare the theoretical results expressed by Eqs. (4) and (6) with our measured results. We use the LCAO results of $W_{d}=11.12 \mathrm{eV}$ and $Z_{d}=4.18$ (Ref. 16) (which implies that $Z_{s}=0.82$ and thus $\left.r_{s} / a_{0}=3.28\right)$ for Ta. With these parameters Eq. (4) predicts $\left(\Delta E_{\text {core }}^{i}\right) /(\Delta a / a)=+5.0 \mathrm{eV}$, and Eq. (6) predicts $\left(\Delta E_{\text {core }}^{f}\right) /(\Delta a / a)=+4.8 \mathrm{eV}$. Combining the initial- and final-state contributions produces a total theoretical shift of $\left(\Delta E_{\text {core }}^{t}\right) /(\Delta a / a)=+9.8 \mathrm{eV}$, where $\Delta E_{\text {core }}^{t}$ $=\Delta E_{\text {core }}^{i}+\Delta E_{\text {core }}^{f}$. Experimental values of $\left(\Delta E_{\text {core }}^{t}\right) /$ $(\Delta a / a)$ are obtained from the slopes of the straight-line fits shown in Fig. 3: for the bulk and surface we obtain values of $+26.5 \pm 2.1$ and $+11.1 \pm 2.0 \mathrm{eV}$, respectively. While the experimental surface value agrees quite well with the $+9.8-\mathrm{eV}$ prediction, the bulk value is significantly larger. We note that the experimental shifts are positive, as expected for a $d$-band metal with $Z_{d}<5$.

Experimental BE changes between 77 and $293 \mathrm{~K}$ can be obtained from the experimentally derived slopes $\left[\left(\Delta E_{\text {core }}^{t}\right) /(\Delta a / a)\right]$ and the value of $\Delta a / a=1.2 \times 10^{-3}$ (Ref. 23) for Ta between these two temperatures. We obtain $+31 \pm 3 \mathrm{meV}$ for the bulk atoms and $+13 \pm 2 \mathrm{meV}$ for the $\mathrm{Ta}(100)$ surface atoms. We also obtain a corresponding thermal shift of $+18 \pm 4 \mathrm{meV}$ for the $\mathrm{Ta}(110)$ surface atoms; this comes from a previous measurement of the decrease in SCS of $13 \pm 2 \mathrm{meV}$ between 77 and $293 \mathrm{~K}$ for $\mathrm{Ta}(110)$ (Ref. 24) and our present result for the bulk shift.

The differences in thermal shifts between bulk and surface atoms of Ta suggest that details of the $d$-electron electronic structure, which have not been considered here, are probably of some importance. For example, surface states likely play a role in thermal shifts of the surface atoms. This inequality in the surface and bulk thermal shifts for Ta is in striking contrast to results on the alkali metals in which, despite the large thermal shifts of $\sim 60 \mathrm{meV}$, no difference was observed between the bulk and surface thermal shifts. ${ }^{4}$

We have used Eqs. (4) and (6) to estimate trends in the thermal change in $\mathrm{BE}$ for all of the $5 d$ transition metals. In the calculations we have used the room-temperature linear expansion coefficient ${ }^{25}$ and the LCAO $d$-electron bandwidths and $s$-band occupation numbers. ${ }^{16}$ The results for $\Delta E_{\text {core }}^{i}, \Delta E_{\text {core }}^{f}$, and $\Delta E_{\text {core }}^{t}$ are plotted in Fig. 5 for a $100-\mathrm{K}$ temperature increase. The figure indicates for metals at the

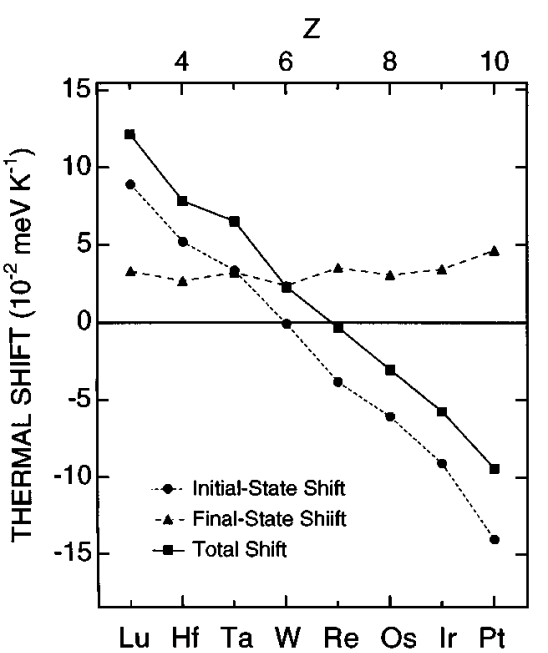

FIG. 5. Model calculation results for thermal core-electron BE shifts for the $5 d$ transition-metal series. Initial state $\left(\Delta E_{\text {core }}^{i}\right)$, final state $\left(\Delta E_{\text {core }}^{f}\right)$, and the total shift $\left(\Delta E_{\text {core }}^{t}\right)$ are displayed for a $100-\mathrm{K}$ increase in temperature.

end of the series that the negative initial-state contribution may dominate the positive final-state contribution and produce an overall negative shift with increasing temperature. Measurements on Pt or Ir would prove most interesting in terms of validating this simple model. Application of the model to the $3 d$ and $4 d$ transition metals also shows a crossover from positive shifts to negative shifts near the middle of each series.

As a last note we mention that very recent temperaturedependent measurements of the $4 f$ core levels of $\mathrm{Lu}$, the first element in the $5 d$ transition-metal series, show thermal shifts between 20 and $293 \mathrm{~K}$ that are smaller in magnitude than 8 $\mathrm{meV},{ }^{6}$ whereas our model predicts approximately $15 \mathrm{meV}$ for such a shift. In contrast to Ta, these measured shifts are smaller than our model predicts.

In summary, we have presented measurements of thermally induced shifts for the $4 f_{7 / 2}$ binding energies of $\mathrm{Ta}(100)$. These results are in reasonable agreement with a simple description that is based upon lattice-expansion driven changes in $d$-band transition-metal electronic structure. Further experimental work on other $d$-band metals is needed to test the proposed description of the shifts, while theoretical work could provide insight into the differences in the shifts between the bulk and surface-atom core levels.

\section{ACKNOWLEDGMENTS}

The authors would like to thank David Peak for a critical reading of the manuscript. This work was supported by NSF Grant No. DMR93-03091 and the Robert A. Welch Foundation (University of Texas). The National Synchrotron Light Source is supported by the DOE Division of Material Science and Division of Chemical Sciences. 
*Present address: Pohang Accelerator Laboratory, Pohang Institute of Science and Technology, Pohang P.O. Box 125, 796-600, Korea.

${ }^{1}$ M. A. Butler, G. K. Wertheim, D. L. Rosseau, and S. Hüfner, Chem. Phys. Lett. 13, 473 (1972).

${ }^{2}$ J. A. Tagle, E. T. Arakawa, and T. A. Callcott, Phys. Rev. B 21, 4552 (1980).

${ }^{3}$ P. H. Citrin, G. K. Wertheim, and Y. Baer, Phys. Rev. 16, 4256 (1977).

${ }^{4}$ D. M. Riffe, G. K. Wertheim, D. N. E. Buchanan, and P. H. Citrin, Phys. Rev. B 45, 6216 (1992).

${ }^{5}$ W. Thesis and K. Horn, Phys. Rev. B 47, 16060 (1993).

${ }^{6}$ E. Weschke, A. Höhr, S. Vandré, C. Schüssler-Langeheine, F. Bødker, and G. Kaindl, J. Electron Spectrosc. Relat. Phenom. 76, 571 (1995).

${ }^{7}$ D. Spanjaard, C. Guillot, M.-C. Desjonquères, G. Tréglia, and J. Lecante, Surf. Sci. Rep. 5, 1 (1985), and references therein.

${ }^{8}$ P. H. Citrin and G. K. Wertheim, Phys. Rev. B. 27, 3176 (1983), and references therein.

${ }^{9}$ R. G. Musket, W. McLean, C. A. Colmenares, D. M. Makowiecki, and W. J. Siekhaus, Appl. Surf. Sci. 10, 143 (1982).

${ }^{10}$ S. Doniach and M. Sunjić, J. Phys. C 3, 285 (1970).

${ }^{11}$ D. M. Riffe, W. Hale, B. Kim, and J. L. Erskine, Phys. Rev. B 51, 11012 (1995).

${ }^{12}$ The structure of the $\mathrm{Ta}(100)$ surface is nearly that of an ideal truncated bcc (100) surface, with a slight contraction $(\sim 10 \%)$ of the first interlayer spacing and perhaps a slight expansion $(\sim 1 \%)$ of the second interlayer spacing. See R. A. Bartynski et al., Phys. Rev. B 40, 5340 (1989), and references therein.

${ }^{13} \Gamma$, which is inversely proportional to the lifetime of the core hole state, and $\alpha$, which describes the high-binding-energy power- law tail of metallic core levels, are temperature-independent quantities.

${ }^{14}$ The effective $\Delta a / a$ for the first atomic layer is likely slightly different from that of the bulk since thermal expansion perpendicular to the surface plane is probabily different from that parallel to the surface plane. However, since we know of no measurements of this perpendicular thermal expansion for the $\mathrm{Ta}(100)$ surface atoms, we use the bulk value of $\Delta a / a$ for both the surface and bulk atoms in discussing the data.

${ }^{15} \mathrm{~J}$. Friedel, in The Physics of Metals, edited by J. M. Ziman (Cambridge, New York, 1969), p. 494.

${ }^{16}$ W. A. Harrison, Electronic Structure and the Properties of Solids (Dover, New York, 1989).

${ }^{17}$ The atomic radius $r_{0}$ is defined by the equation $V_{\text {atom }}=\frac{4}{3} \pi r_{0}^{3}$, where $V_{\text {atom }}$ is the volume/atom in the solid.

${ }^{18}$ Equation (3) is derived under the assumption that there is no charge transfer between the $s$ and $d$ bands as the lattice expands. Relaxing this assumption alters the results for Ta by less than $4 \%$. Hence the effects of $s$-band to $d$-band charge transfer can be neglected.

${ }^{19}$ L. Hedin and S. Lundqvist, in Solid State Physics, edited by F. Seitz, D. Turnbull, and H. Ehrenreich (Academic, New York, 1969), Vol. 23, p. 1.

${ }^{20}$ N. Singh and S. P. Singh, Phys. Rev. B 41, 12509 (1990).

${ }^{21}$ R. E. Watson, M. L. Perlman, and J. F. Herbst, Phys. Rev. B 13, 2358 (1978).

${ }^{22}$ A. R. Williams and N. D. Lang, Phys. Rev. Lett. 40, 954 (1978).

${ }^{23}$ Thermophysical Properties of Matter, edited by Y. S. Touloukian (Plenum, New York, 1970).

${ }^{24}$ D. M. Riffe and G. K. Wertheim, Phys. Rev. B 47, 6672 (1993).

${ }^{25}$ A. J. Moses, The Practising Scientist's Handbook (Van Nostrand Reinhold, New York, 1978), pp. 580-684. 